\title{
Quaderni
}

QUADERN I Communication, technologies, pouvoir

\section{Profits symboliques et identité(s) : les think tanks entre affirmation et dénégation}

\author{
Lucile Desmoulins
}

\section{(2) OpenEdition}

\section{Édition électronique}

URL : http://journals.openedition.org/quaderni/503

DOI : 10.4000/quaderni.503

ISSN : 2105-2956

\section{Éditeur}

Les éditions de la Maison des sciences de l'Homme

\section{Édition imprimée}

Date de publication : 5 octobre 2009

Pagination : 11-27

\section{Référence électronique}

Lucile Desmoulins, «Profits symboliques et identité(s) : les think tanks entre affirmation et dénégation », Quaderni [En ligne], 70 | Automne 2009, mis en ligne le 05 octobre 2011, consulté le 02 mai 2019. URL : http://journals.openedition.org/quaderni/503 ; DOI : 10.4000/quaderni.503 


\section{$D$ ossier}

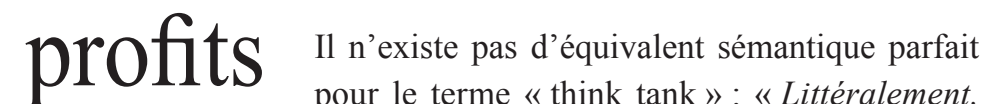
pour le terme «think tank »: "Littéralement,

symboliques et identité(s) :

les think tanks

entre affirmation

et dénégation

\section{Lucile Desmoulins}

Maître de Conférences

Université Charles de Gaulle Lille III Membre du GERIICO think tank signifie «réservoir intellectuel» ou, pour utiliser un anglicisme, "boîte à penser» 》 (Béland, 2000 : 253). Les traductions les plus courantes sont les suivantes : « institut de recherche » «laboratoire d'idées », mais aussi « cercle de réflexion », et «boîte à idées ». Force est de constater que les références aux think tanks dans l'espace public sont pourtant légion (discours politiques et articles de presse) et incitent à questionner la place et le rôle joué par les organismes qui se réclament de cette dénomination ou qui la voient attribuée dans le champ politico-médiatique. Cet article se propose d'appréhender la greffe du modèle d'expertise incarné par les think tanks à travers une approche communicationnelle à partir du cas des think tanks français ayant émergé à compter des années 1970 et s'intéressant aux questions internationales et de défense ${ }^{1}$.

Nous analyserons tout d'abord les enjeux politiques afférents aux débats relatifs à la définition du concept de «think tank », les profits symboliques et l'effet de labellisation dont les think tanks bénéficient. Dans un deuxième temps, nous envisagerons les outils de communication des think tanks et les efforts des chercheurs pour s'approprier de nouvelles ressources d'influence en distinguant le canal de l'expertise politicoadministrative de celui des interventions dans les médias. Ce faisant, nous mettrons en évidence un processus circulaire de légitimation au sein duquel le rattachement à un think tank s'impose comme un « titre à parler » (Tavernier, 2004). Le système d'action de l'expertise politico-médiatique a ainsi fait émerger un groupe hétérogène d'instituts de recherche et d'experts ayant noué 
avec les membres du milieu décisionnel central et les médias des liens ambigus entre dénégation, reconnaissance, instrumentalisation et valorisation.

\section{Think tank : une dénomination, des conno- tations et un label}

Une définition ambiguë propice à l'instrumentalisation

On ne connaît pas de définition consensuelle pour le concept de think tank. Ces organismes existent souvent sous des formes hybrides. Pour analyser l'expertise internationaliste et stratégique française au regard du modèle incarné par les think tanks, une définition rigide s'est révélée stérile. Seule une définition très peu discriminante nous a paru inattaquable: «institutions de recherche en politiques publiques à caractère non lucratif et jouissant d'une autonomie organisationnelle substantielle» (Hames, Feasey, 1994 : 216). Cette définition présente l'avantage de ne pas donner prise à des interprétations idéologiques quant au rôle politique et social des think tanks.

Victimes des connotations laudatives associées à la dénomination, les think tanks se voient intégrés à des théories sur l'État. Plaidant en faveur d'une approche en termes de pouvoir, certaines défendent l'idée de think tanks rhétoriciens, véritables chambres d'écho au service de l'élite dominante. Les think tanks participeraient à un réseau homogène d'élaboration des programmes politiques avec les fondations, les facultés les plus prestigieuses, les journalistes, les dirigeants d'entreprises (Domhoff, 1971 : 213). Aux antipodes de cette vision marginale de think tanks instruments de contrôle au service de l'élite économique, on identifie nombre de travaux les présentant comme une preuve de pluralisme accru, de l'avènement d'un monde plus démocratique car davantage marqué par l'intervention de la société civile dans les processus de décision politique. Tout comme les ONG ont été perçues comme des «signes avant-coureurs d'un monde plus libéral et bienveillant » (Cohen, 2003 : 53), les think tanks seraient les primus inter pares de la société civile selon la théorie du pluralisme, des «phares » pour une société civile mobilisée et informée. Cette vision du rôle messianique des think tanks est très prégnante dans leur communication externe. Dans le texte de présentation du site Internet de l'IFRI, par exemple, on peut lire: "Centre de rencontres et de débats, l'Ifri favorise une interaction fructueuse entre les administrations, le secteur privé, la communauté internationale des chercheurs et les représentants de la société civile au cours d'échanges de vues avec acteurs ou observateurs qualifiés de la scène internationale $»^{2}$. Les think tanks seraient de surcroît les preuves tangibles de l'ouverture des circuits décisionnels à des sources d'information et d'analyse extra administratives d'où une rationalisation des processus de décision. D'autres auteurs évoquent les think tanks en terme de fluidification de la circulation des idées dans un contexte de mondialisation. Ces derniers permettraient une intégration culturelle rapide à l'échelle mondiale des mêmes catégories d'analyse et visions du monde. La prolifération du modèle des think tanks serait ainsi la garantie d'une diffusion du modèle démocratique. Le « discours hégémonique (Haubert et al., 2000) sur la société civile en tant que contre-pouvoir nécessaire à l'État rencontre un discours de lé- 
gitimation des think tanks en tant qu'instigateurs de la démocratisation ou bien en tant que garantie d'ouverture et de rationalisation des processus de décision. Toutes ces théories ne résistent pas à une étude approfondie de la greffe du modèle des think tanks en France.

Avant d'étudier les think tanks français, il convient de se positionner vis-à-vis des travaux qui postulent l'incomparabilité des think tanks anglo-saxons et accréditent l'idée d'une exception française en matière de think tanks. Thierry de Montbrial décrit l'IFRI comme « le seul think tank français $»^{3}$. Évoquer des think tanks français, c'est d'ailleurs s'attirer systématiquement des réflexions dubitatives ou amusées : «Vous croyez vraiment que l'on peut parler de think tanks français? $»^{4}$. Ces réflexions s'intègrent bien dans l'idée très répandue que le système politique français fait exception. Cette approche exceptionnaliste a pu conduire au rejet de la comparaison par « respect du contenu historique des objets analysés » (Badie, Hermet, 1990 : 20) et à la conclusion suivante : les modèles français et américain d'institutionnalisation de l'expertise sont stables, irréductibles et incomparables et les instituts français de recherche sur des questions internationales et stratégiques ne sont pas de vrais think tanks. Dans cette lignée, certains chercheurs comparent les think tanks britanniques avec les cabinets ministériels français (Gaffney, 1996) ou assimilent les clubs politiques français à des think tanks en s'appuyant sur le concept de « communauté épistémique » (Fieschi, Gaffney, 1998). À l'instar de Diane Stone, James McGann, Martin Thunert et Donald Abelson, nous reconnaissons l'exemplarité du modèle anglo-saxon liée au financement philanthropique des think tanks, mais plaidons pour une prise en compte des spécificités nationales. Les études portant sur la transnationalisation des think tanks témoignent, en effet, du caractère hybride, fragile et contesté de la forme think tank de l'expertise dans les pays mêmes qui l'ont vue naître. À titre d'exemple, en Grande-Bretagne et aux États-Unis, on confond de plus en plus operating foundations ${ }^{5}$, think tanks, ONGs et lobbies.

De nombreux think tanks français ont été créés en référence explicite aux plus célèbres think tanks américains (Rand Corporation, Brookings Institution, Council on Foreign Relations) ou britanniques (RIIA) suivant un processus mimétique d' « import-export institutionnel » (Rouban, 1993). À l'instar de James G. McGann (1995)', nous estimons savoir que nous avons affaire à un think tank quand nous en avons un sous les yeux. Consciente que la définition « n'est pas le privilège du chercheur. Elle renvoie aussi à des référentiels propres aux objets et aux activités étudiés » (Werner, Zimmermann, 2004 : 30), voici les éléments que nous retenons pour caractériser un think tank :

- une structure organisationnelle stable (pérennité assurée, fonction support administrative, centre de documentation, bibliothèque, bureaux) ; un personnel de recherche salarié et pluridisciplinaire au niveau de compétence et de recrutement élevé (doctorat) ; le confort des ressources financières et la variété de leur provenance car un think tank idéal ne dépend pas d'un seul bienfaiteur ou client ;

- la recherche comme activité primordiale, devant les missions d'expertise politico-administrative et la communication auprès d'un public élargi 
(publications, conférences, enseignements, interviews, articles) ;

- la capacité réalisée de produire des analyses de fond, des travaux de vulgarisation et des propositions politiques directement applicables à destination des décideurs ; une politique de diffusion des travaux et de communication diversifiée (édition d'ouvrages, de revues, interventions dans les médias sur le mode de l'interview, des articles et des citations, séminaires de formation, réunions, colloques, interventions lors des conférences internationales);

- la reconnaissance des médias qui favorise la publicisation; la participation à des réseaux de chercheurs et de décideurs favorisant l'accès aux membres des milieux décisionnels centraux.

Dans la pratique, l'ensemble de ces critères n'est jamais respecté. Il n'existe pas de think tank pur et parfait, ni en France, ni aux États-Unis. Au sein du paysage hétérogène de l'expertise internationale et stratégique, nous nous sommes limités aux plus grands centres de recherche intervenus régulièrement dans des processus d'expertise à travers des contrats d'études réguliers ou des conventions d'études avec la DAS (ministère de la Défense) et/ou le CAP (ministère des Affaires étrangères) : l'IFRI, la FRS, le CERI, et l'IRIS'. Ces quatre centres bénéficient, de surcroît, d'une bonne visibilité et reconnaissance médiatique.

Un effet de labellisation de la dénomination dans les médias français

Flou dans sa définition, le concept de think tank se prête à des jeux de représentation et opérationalise des enjeux d'instrumentalisation. Paul Dickson donne en exemple divers articles de presse montrant que, dans les années soixantedix, le public voyait encore les think tanks comme des sortes de conseillers bénévoles omnipotents, capables de résoudre « tous les petits problèmes du quotidien et les gros problèmes de la société » (Dickson, 1971 : 28). Il en est de même en France aujourd'hui. Des connotations principalement laudatives sont associées aux think tanks par la plupart des acteurs du processus décisionnel et des médias. Dans la presse, l'expression anglaise est conservée et parfois traduite. Elle est flatteuse en vertu de la prime réservée aux anglicismes. La perception française des think tanks est, en effet, marquée par une conception plus globale des États-Unis, le pays qui a vu naître l'appellation et qui est porteur «de fascinations et d'aversions » (Lacorne, Rupnik, Toinet, 1986). Une impression très favorable au sujet des think tanks anglo-saxons ressort, par exemple, d'un rapport d'information de l'Assemblée Nationale : «Il y a, par ailleurs, $[$...] les Think Tanks ("réservoirs de pensée »), institutions privées, sans définition officielle, pluridisciplinaires, non partisanes, sans but lucratif et en principe indépendantes, [...] Rassemblant des experts, [...] ils alimentent fréquemment les parlementaires en analyses et réflexions au travers de travaux diffusés le plus largement possible et qui bénéficient d'ailleurs d'une audience considérable» (Tron, Deniau, 2003).

Dans les trois principaux quotidiens nationaux français ${ }^{8}$, l'expression think tanks est utilisée accolée avec le nom d'un centre basé dans un pays anglophone, puis elle est utilisée pour décrire des clubs politiques français (Fondation pour l'innovation politique), des groupes de réflexion (Institut Montaigne), des cellules administratives 
(CAE, le Plan $\left.{ }^{9}\right)$. Le terme think tank est massivement associé à un adjectif qualificatif laudatif : «des Liberal democratic studies, un think tank très réputé $»^{10,}$ "le très droitier institut Hoover, excellent «thinktank», règne »" ${ }^{\prime \prime}$, [i1] dirigera le Aspen Institute, un prestigieux centre de réflexion (think tank) $»^{12}$, « des instituts de recherche, les fameux think tanks $»^{13}$. Incarnation d'une forme d'excellence de la pensée, les think tanks sont aussi perçus comme des acteurs incontestablement influents sur la scène politique : «Il est difficile de dire dans quelle mesure l'influence de nos hommes fut positive ou négative, mais en tout cas leur influence fut considérable. » (Dickson, 1971 : 90) ${ }^{14}$. Leurs travaux seraient largement diffusés, lus avec attention et considérés avec le plus grand respect.

Diane Stone a délimité le périmètre des think tanks de manière très parlante en s'appuyant sur une description de ce qu'ils ne sont pas : des clubs politiques, des lobbies d'affaires, des entreprises de conseil, des fondations (Stone, 1996). Reste que des petites structures américaines variées utilisent le terme pour «faire important » (Dickson, 1971 : 28). En France, les discours d'autocélébration légitimante procèdent d'enjeux de visibilité et d'identité d'où des affirmations d'indépendance, de non lucrativité, de transparence, de scientificité, d'influence, etc. Dans la mesure où la dénomination est mal comprise, certains lobbies français se comparent à des think tanks pour gommer le caractère agressif de leurs méthodes et leurs partis pris incompatibles avec la démarche scientifique : « nous sommes un think tank efficace ». Plus étrange, la dénomination think tank a été revendiquée par, ou attribuée à une cellule de réflexion et de prospective intra- administrative rattachée à Matignon (le Conseil d'Analyse Économique ${ }^{15}$ ), à des clubs politiques (la Fondation pour l'innovation politique ${ }^{16}$, un club rattaché à l'UMP), à des groupes de réflexion internes à un état-major de campagne ${ }^{17}$, à des ONG militantes ${ }^{18}$ et à des entreprises de conseil (The Economist Intelligence Unit ${ }^{19}$, la branche 'études économiques' d'un grand groupe de presse). Le caractère mélioratif de l'appellation «think tank» s'incarne dans le complexe d'infériorité de certains décideurs politiques, hauts fonctionnaires, officiers militaires et membres de think tanks français qui dénoncent ou s'interrogent sur l'emploi en France de la dénomination: "Il n'y a pas de think tanks en France », "Vous-même, vous pensez avoir réussi à cibler un objet? ", « Ce sont les journalistes qui disent que nous sommes un think tank». Les connotations péjoratives associées aux think tanks sont rares au point d'être négligeables.

Les journalistes ont bel et bien joué un rôle-clé dans l'effet de labellisation des think tanks. Les think tanks apparaissent dans la presse française sur le mode de l'interview d'experts ou du style indirect, c'est-à-dire de la parole d'experts insérée à titre de caution scientifique, à l'intérieur de textes rédigés par des journalistes. Les interviews de chercheurs sont devenues la caution scientifique des médias, la béquille des journalistes. Les think tanks sont des sources de prédilection pour des journalistes sommés de produire des analyses sur un large panel de sujets inégalement maîtrisés. Dans les cas de style indirect, de parole rapportée, ce n'est pas le nom du chercheur qui est mis en avant, mais le nom du think tank qui sert de caution aux propos du chercheur partiellement retranscrits. "Monsieur Smith, chercheur de 
la Rand Corporation, un prestigieux think tank américain de la côte ouest affirme que ", est l'exemple-type de cette forme d'intervention. Parfois, le think tank est même personnifié, il pense, affirme, décide, encourage, dénonce. Les think tanks anglo-saxons comme français s'imposent ainsi en tant que sources d'information et d'analyse incontestables. Dans la presse, l'emploi du terme think tank correspond aussi à une facilité d'écriture de journalistes hésitant quant à l'identité de certaines des institutions auprès desquelles ils puisent des informations.

Des stratégies de communication externe imprégnées par une recherche de labellisation

Désireux de professionnaliser leur communication externe, les think tanks ont fait appel à des entreprises privées pour la confection de leur plaquette ou le remaniement de leur logo. Cette aide extérieure a eu pour effet de lisser ces outils de communication et, ce faisant, elle a amoindri leur intérêt heuristique. Internet est intéressant en ce qu'il a changé les règles du jeu de l'influence en abaissant le « ticket d'entrée » dans le monde de la visibilité. L'ambition d'une communication externe labellisée en tant qu'homologues français des think tanks anglo-saxons se donne à voir dans les sites Internet des think tanks français et, a fortiori, leurs premières versions mises en ligne. Les quatre centres étudiés sont entrés presque simultanément dans une réflexion sur l'opportunité de réaliser un site Internet et de le mettre en ligne, sur les objectifs d'un tel site et son contenu ${ }^{20}$. La mise en ligne de leurs sites a répondu à un souhait de communiquer vers de vastes publics, mais aussi à une logique de concurrence entre think tanks français pendant une période d'effervescence liée à la bulle spéculative Internet. Ces sites ont été créés sur le modèle des sites Internet des think tanks américains et britanniques les plus prestigieux, ils ont aussi évolué de manière incrémentale en fonction d'une veille concurrentielle. Ils ont tous été réalisés et réactualisés grâce à des moyens modestes, par tâtonnements et en interne.

À travers le choix des données proposées à la lecture, leur présentation, l'organisation de l'espace visuel, l'agencement des cadres, leur hiérarchie, le calibrage et l'emploi des liens ou « signes passeurs », se dévoile l'énonciation éditoriale des écrits d'écran des think tanks, leurs intentions de communication. L'indéfinition de leur rôle apparaît, par exemple, dans le flou et la diversité des destinataires potentiels de leurs sites Internet : membre du milieu décisionnel central ou grand public, agents ministériels ou homologues étrangers, chercheurs, entreprises, étudiants, grand public. De même, un discours très revendicatif illustre leur besoin de reconnaissance, leur difficulté à gérer des conflits identitaires du fait de l'indéfinition de leur rôle social.

L'homogénéité du discours communicationnel des think tanks étudiés est révélatrice d'un souci et d'une ambition communs de pérennité, de visibilité et de légitimité. Par exemple, tous ces sites témoignent d'une recherche de labellisation illustrée par l'omniprésence de leur logo et de la dénomination think tank. Cette communication de marque témoigne d'une quête de notabilité et de légitimité. Via leurs sites Internet, les think tanks français augmentent leur visibilité institutionnelle sur la scène politico-médiatique plutôt qu'ils n'augmentent leur capacité d'influencer. 
Les domaines de compétences ou axes de recherche, par exemple, ne se donnent pas à voir immédiatement, ils se dévoilent après un surf prolongé dans ces sites. En outre, ces sites ne sont que peu dédiés à la diffusion d'informations et d'études de fond. Deux registres de discours cohabitent nettement, le premier est axé sur l'institution et le deuxième, sur ses productions et leurs destinataires. Le discours institutionnel autoréférentiel est une forme de mise en scène tournée vers le statut de think tank. L'IFRI, par exemple, se présente à travers les photos de son site comme un prestigieux lieu de rencontres et de représentation diplomatique (architecture du bâtiment) et comme le centre névralgique d'un réseau d'entreprises (respect scrupuleux des codes de la communication corporate).

La représentation limitée des chercheurs en tant qu'individus est symptomatique d'une opposition entre les deux niveaux du collectif et de l'individuel. La difficulté des think tanks à exister sur la scène politique et dans les débats publics en tant qu'institutions collégiales est matérialisée par la figuration sur un mode mineur des chercheurs qui les composent, par rapport à d'autres informations valorisant la dimension collective (colloques). Les think tanks se donnent à voir à la fois comme instituts de recherche et comme maisons d'édition. Cette double posture leur permet d'exister de manière simple et convaincante, à travers des images scannées de couvertures de livres ou de revues. En tant qu'éditeurs, ils accroissent leur légitimité vis-à-vis de leurs commanditaires et bienfaiteurs (administrations, entreprises et fondations). Ils améliorent leur crédibilité académique vis-à-vis des journalistes et confèrent de la visibilité à une activité «ésotérique » aux yeux du grand public : la recherche. Cependant, la résorption d'une tension majeure paraît impossible, entre une labellisation collégiale des productions telle qu'elle existe aux États-Unis où l'on parle d'une «étude Brookings », et le caractère individuel des activités et des ambitions des chercheurs.

\section{Les supports et outils d'information et de communication des think tanks français : un double circuit de légitimation expertal et médiatique}

L'émergence de think tanks à la française a bénéficié du développement de procédures d'expertise pour le compte de la Délégation aux affaires stratégiques (DAS), inaugurée en 1992 au sein du ministère de la Défense, et du Centre d'analyse et de prévision (CAP), créé en 1973 au sein du ministère des Affaires étrangères. Ces deux administrations jouent aujourd'hui un rôle-clé de passerelle entre les deux mondes de la recherche et de la décision. Elles partagent les mêmes missions : produire et fournir une analyse des grands problèmes internationaux ayant trait à la politique étrangère et de sécurité de la France. Non seulement elles réalisent des études en interne, mais encore elles externalisent cette recherche d'informations et d'analyses à travers des contrats d'expertise passés avec des centres de recherche. Elles maîtrisent la formulation d'une question, la procédure de mise en concurrence des mandataires, le pilotage et les modalités de publicisation des résultats des études. Grâce à ces administrations, un réseau de chercheurs-experts a pu émerger. Ils sont à la fois des « marginaux sécants » (Jamous, Commaille, Pons-Vignon, 1969) et des « navigateurs organisationnels » (Pelletier, 
1995) capables de traverser la frontière qui sépare la décision et la recherche bien que la France ne connaissent pas le système des revolving doors. Leur bilan positif doit être nuancé. La fonction des contrats de recherche s'avère plus complexe que la simple captation d'informations et d'analyses élaborées en toute indépendance à partir de connaissances et de méthodes scientifiques. Les deux dispositifs procéduraux de l'expertise internationaliste et stratégique étudiés ne sont pas considérés comme un canal d'influence efficient et les chercheurs identifient moins l'expertise comme un répertoire d'action, que comme un système officieux de subventions. La notion d'expertise s'applique mal aux relations entre think tanks et administrations françaises, car on y observe rarement « la rencontre d'une conjoncture problématique et d'un savoir spécialisé» (Tanguy, 1995).

L'étude comparée de deux formes d'énonciation de l'expertise est marquée par leur caractère diamétralement opposé. Les notes CAP se rapprochent des codes sémiotiques des notes de synthèse administratives, et les rapports DAS reprennent les formes scripturales d'exposition de la recherche, et notamment celles des objets « thèse » et « mémoire ». Ces deux dispositifs de médiation peuvent être rapprochés en ce qu'ils témoignent d'une intériorisation d'attentes politiques et d'un effort des chercheurs pour influencer favorablement la réception par les commanditaires des travaux d'expertise.

Tous les chercheurs sont acculés à une réflexion sur leur communication, ils doivent assumer un rôle de médiateur de leur savoir et redéfinir leur mode de production et de diffusion de connais- sances. Dans certaines monographies anglaises, les think tanks sont d'ailleurs décrits comme des traducteurs, vulgarisateurs, marketeurs d'idées produites ailleurs. Cette posture suppose de la part des chercheurs une forme de modestie intellectuelle quant à la force de conviction de leurs seules analyses et la recherche d'une « proximité langagière » (Roy, 2001) lors de leurs rencontres avec des membres du milieu décisionnel. La rhétorique de l'expertise s'apprend par tâtonnements au fur et à mesure des expériences de franchissement des frontières institutionnelles. En intégrant la communauté des experts parlant depuis un think tank, les chercheurs sont formés à l'expertise, ses codes, ses contraintes, ils « apprennent [...] les «métacodes» des univers situés au-delà de leur milieu d'appartenance » (Pelletier, 1995).

L'effort rhétorique réalisé consiste principalement en une priorité accordée aux registres de l'opérationnalité et de la prospective et en une pédagogie ludique des représentations (Migne, 1969) : comparaisons, métaphores, illustrations. Il concerne une simplification du discours et suppose la présence accrue de synthèses, l'abandon des nuances au profit d'énoncés catégoriques. Les chercheurs estiment «favoriser un discours binaire », emprunter des tournures aux sciences dures ( "l'équation de la paix») et des phrases ou expressions-choc («complicité de génocide»). Des figures rhétoriques comme l'analogie, la comparaison, le néologisme (Jacobi, 1986) ont mauvaise presse auprès de chercheurs. Ceuxci déplorent "la passion des listes à puces», «la manie des encadrés », «les présentations powerpoint à tout bout de champ », l'emploi obligé de clichés, d'expressions caricaturales, 
lyriques, passéistes ou normatives porteuses d'erreurs d'analyse. Pourtant, ils les emploient car elles agissent comme des accélérateurs cognitifs. Certains chercheurs valorisent cet effort de vulgarisation comme une compétence supplémentaire : "Je sais marketer une étude afin de maximiser sa diffusion dans les circuits politico-administratifs ».

En théorie, deux stratégies ou postures contradictoires sont à la disposition des chercheurs en situation d'expertise. La première concerne la modération : choix de présenter des informations arrangeantes, de formuler ses analyses de manière à ce qu'elles ne heurtent pas le commanditaire. La deuxième relève au contraire de la «scandalisation » (Rieffel, Neveu, 1991) : choix de jouer sur le registre de l'intelligibilité (mise en avant des éléments les plus critiques de l'analyse) ou de l'émotion (mise en scène dramatique). De manière générale, les discours de l'expertise sont très modérés. Le recours à la « scandalisation » s'opère dans l'espace médiatique plutôt que celui de l'expertise. Les procédures d'expertise incitent à la conformité aux attentes anticipées du commanditaire, à la « maîtrise du discours convenable, selon les critères de convenance propres à la communauté dont on attend l'approbation » (Gardin, 2004). Il s'agit de faire passer des idées, mais aussi de ménager le commanditaire afin de préserver ses ressources financières futures. Seuls les chercheurs ayant atteint un niveau très élevé de reconnaissance peuvent «se payer le luxe de ruer dans les brancards ». De même, les chercheurs dénoncent les intitulés des études et les consignes formulées par les agents de la DAS qui les pilotent. Pourtant, ils s'y conforment validant l'hypothèse d'une forme « d'hypocrisie commu- nicationnelle ». Les trois conditions ${ }^{21}$ qui constituent le cadre normatif de la discussion ne sont que rarement remplies dans le cadre de l'expertise politico-administrative : la symétrie, la sincérité et la liberté d'adhésion (Habermas, 1999).

Dans la mesure où les dispositifs scripturaux d'expertise sont en déshérence, le principe de rationalité dialogique intervient principalement dans les situations de communication en paroles. Les réunions d'avancée et de restitution des travaux sont donc perçues comme des espaces privilégiés de communication, que ce soit lors de réunions de pilotage en tête-à-tête ou de réunions formelles de restitution d'une étude. Ainsi, les rencontres humanisent la relation d'expertise, elles viennent contredire les clichés et les dénigrements basés sur des considérations purement statutaires. La compréhension viendrait d'une interaction langagière qui s'épanouirait idéalement tout au long du processus d'expertise par capillarité et atteindrait son faîte lors de la réunion de restitution. Des qualités d'élocution, de clarté du discours, mais également le charisme ou l'adaptation au contexte de communication d'une relation de type commerciale favorisent la rencontre. Les décideurs ont ainsi mentionné les noms de chercheurs «qui en imposent», "qui déplacent les foules », "Il passe bien. Il fait ni trop long, ni trop court»; "Il répond bien aux questions, sans tourner autour du pot ». La «dimension paralinguistique » de la communication n'est pas en reste (Mucchielli, 1997 : 41). Les règles de l'interaction d'expertise sont aussi vestimentaires : «Les Ingénieurs de l'Armement mettent un costume, ça a l'air idiot, mais j'ai acheté un costume, c'est un moyen de ne pas être catalogué comme étant l'universitaire qui 
se distingue en ne mettant pas de costume ». La discussion a souvent été rendue possible dans des contextes de crise aiguë ayant motivé, du côté des administrations et des décideurs politiques, une recherche d'informations tous azimuts.

Reste que le statut des rencontres entre experts et décideurs varie à l'extrême, de la discussion dialogique à l'échange formel de pure convenance. L'échange oral n'a parfois pas lieu, par indifférence, désintérêt ou manque de temps. Le modèle de la longue discussion vive, nourrie et respectueuse n'est en rien généralisable. Lors des rencontres entre une administration commanditaire et le membre d'un think tank mandataire, la rationalité des situations d'expertise n'est pas forcément dialogique, elle peut être processuelle et procédurale, instrumentale ou dramaturgique. Dans le premier cas, le commanditaire d'une étude agit alors en gestionnaire, il vérifie que la somme dépensée soit concrétisée par l'obtention d'un objet matériel : un rapport, une note, ou une réunion. L'échange se situe parfois principalement à un niveau de rationalité instrumentale. En ce cas, l'intérêt n'est pas porté au contenu de l'étude, mais à l'échange de légitimité. Enfin, la rationalité de l'échange est souvent dramaturgique. Le chercheur et le décideur n'échangent jamais que de manière codifiée dans le souci de se conformer à l'image qu'ils ont de leur statut. Ils jouent alors un rôle appris par cœur comme sur la scène d'un théâtre. Chacun exprime des points de vue en fonction de l'idée qu'il se fait de son identité professionnelle et de l'éthique qu'il lui rattache. Le chercheur se drape dans une position morale lui interdisant de se positionner ou de s'engager, d'émettre des propositions opérationnelles et stratégiques, il se fait l'exégète de la complexité, redoute les ten- tatives d'instrumentalisation ou de musellement. Le décideur lui renvoie son accès à des sources d'information discordantes, son emploi du temps surchargé, sa marge de manœuvre limitée. La communication est de forme diptyque. Chacun dit sa vision du monde et les différents discours sont des monologues qui s'épanouissent de manière parallèle, sans jamais se rejoindre (Plantin, 2004). La déshérence des dispositifs et des discours d'expertise a poussé les experts rattachés à un think tank à un investissement très volontaire de stratégies discursives médiatiques.

\section{La légitimation médiatique des experts parlant depuis un think tank}

L'investissement de stratégies discursives médiatiques est favorisé par le rattachement de certains chercheurs à un think tank car ceux-ci ont valeur de « titres à parler ». Les paroles d'experts dans les médias participent à un processus circulaire de légitimation entre les décideurs, l'opinion publique, les médias et les think tanks. Un système de relations et d'échange de légitimité rassemble journalistes et chercheurs. Sur le fond, les chercheurs attirent l'attention de l'opinion publique en faisant appel à des stratégies de «scandalisation» qui exploitent le sentiment d'empathie (ex. : théâtralisation de la souffrance humaine), ou qui déplacent les débats politiques vers un niveau éthique. Des experts ont ainsi pu se transformer en leaders d'opinion ou bien s'adjoindre le service de leaders d'opinion (intellectuels, responsables d'ONG, éditorialistes, etc.). Certains sont même allés jusqu'à endosser le costume du militant ou de l'éminence grise dans l'ombre d'un parti politique pour exercer une « capacité de nuisance » jugée plus efficace 
que leur capacité d'analyse.

Si les chercheurs appartenant ou rattachés à un think tank sont très sollicités par les journalistes, c'est tout d'abord parce qu'ils possèdent un « titre à parler » et ensuite parce qu'ils maîtrisent les règles pragmatiques et sémantiques du discours qui satisfont aux interventions médiatiques au titre d'expert. Si les situations d'expertise politico-administrative supposent une adaptation rhétorique, les situations d'expertise médiatique sont encore plus exigeantes. Les chercheurs rattachés à un think tank ont appris à adapter le format et le style de leurs articles aux journaux et aux magasines visés. Ils savent gérer leur temps de parole, ce qui est crucial dans les émissions radiophoniques, et adopter un savoir-être propice à susciter des invitations sur des plateaux de télévision. La recherche « produit peu de personnalités médiatiques » (Tristani-Potteaux, 2001) capables de théâtraliser leurs interventions. La liste des interventions médiatiques des chercheurs figure significativement dans le rapport d'activité de chacun des think tanks internationalistes français. Participer à des émissions de télévision et de radio en tant qu'expert fait partie des devoirs et des compétences des chercheurs rattaché à un think tank, obligation qui les distingue des universitaires. La publication d'un article dans l'un des principaux quotidiens nationaux permet de diffuser des idées autant que de « manifester son importance » (Rieffel, 1993 : 575) au sein de son think tank de rattachement et de faire connaître cet organisme à l'externe. De même, la participation à des émissions de télévision est un réel « adjuvant de notoriété » (Rieffel, 1993 : 596).

On observe une congruence des intérêts des médias et des experts. Les médias se valorisent à travers l'autorité d'une figure de compétence contenue implicitement dans la mention du nom d'un institut de recherche. Les think tanks existent de manière collégiale, ils deviennent le titre à parler ultime, supérieur dans le processus de légitimation à toutes autres dimensions : le diplôme ${ }^{22}$, la discipline académique, le niveau hiérarchique, ou le domaine de compétence. Il est frappant qu'un chercheur soit souvent présenté comme un simple «membre » d'un think tank. Les chercheurs se présentent à travers leur simple appartenance à un collectif prestigieux et s'expriment dans l'espace médiatique avec la force d'un soutien institutionnel.

\section{Conclusion}

Dans un contexte de confinement en interne des débats au sein des administrations, de centralisation des décisions et d'absence de culture philanthropique, la création de think tanks en France a tenu de la gageure. Leurs débuts modestes doivent beaucoup à des arrangements politiques, de même qu'à l'impulsion de personnalités hors du commun, qui ont su s'imposer par leur charisme et leur réseau. Si l'existence des think tanks français a pu évoluer de la précarité à la pérennité, leur rôle reste indéfini, entre réflexion théorique, recherche fondamentale, et apport opérationnel, entre enrichissement des débats publics et proposition de politiques publiques applicables, entre conformité aux référentiels dominants et analyse critique.

Les think tanks sont aussi des fournisseurs d'armes d'une grande technicité : les idées, les concepts et les paradigmes d'analyse. L'hypothèse 
optimiste consisterait à les décrire comme des organisations au «potentiel inaccompli». Une hypothèse pessimiste les observerait davantage en termes d'organisations en lutte pour leur survie institutionnelle, leur visibilité et leur légitimité. Des institutions et des procédures ont été créées, mais il ne s'en est pas suivi de changement majeur en ce qui concerne les processus décisionnels dans le domaine de la politique extérieure de la France, la «boîte noire » de la décision. À de rares exceptions près, le contenu des décisions n'a pas été modifié suite à des interventions de chercheurs en sciences sociales ou de think tanks, que ce soit par le biais de procédures d'expertise, de pressions médiatiques ou de consultations informelles liées à des relations personnelles. L'influence des think tanks et des experts français sur la politique extérieure de la France est restée limitée à un nombre infiniment restreint de décisions. Les exceptions identifiées sont liées à l'ouverture de fenêtres d'opportunités lors de l'imposition impromptue d'un dossier sur l'agenda du fait de situations d'urgence politicomédiatique.

Les dispositifs et les discours de l'expertise incitent à analyser cette dernière comme un avatar et un alibi de l'évaluation. L'expertise serait un discours de légitimation du décideur vis-à-vis des citoyens et de lui-même, un horizon argumentaire qu'il peut opposer aux critiques. Ainsi, les mythes relatifs à l'expertise sont générateurs d'attentes de rôle souvent déçues et de malentendus entre décideurs et chercheurs. En effet, les registres et les injonctions paradoxales y abondent : la proximité, le contrôle et l'indépendance, la prospective et l'opérationnalité, la complexité et la vulgarisation scientifiques, la neutralité et l'engagement, l'originalité et l'acceptabilité. Les décideurs français ont de fait oscillé entre la tolérance polie vis-à-vis des think tanks et leur promotion en tant qu'homologues et interlocuteurs des think tanks américains ou européens. Au-delà de la figure du décideur soucieux d'être bien informé et du fantasme de l'expert capable de résorber la complexité de l'action publique, le mythe de l'expertise s'avère bien écorné. Cette activité, ses dispositifs, ses discours et ses situations sont des lieux privilégiés d'observation des «masques du pouvoir » (Charaudeau, 2005). C'est sur un mode binaire de l'affrontement / dénégation et de l'instrumentalisation / valorisation que se nouent les relations entre politiques et chercheurs en situation d'expertise.

L'expertise internationaliste et stratégique s'est épanouie sous la forme des think tanks qui jouent donc un rôle complexe. C'est du côté de médias en recherche permanente de clés de lecture de l'actualité et de cautions scientifiques que les think tanks français ont le mieux réussi à s'imposer comme des sources d'information et d'analyse. Dans la presse française, la référence aux think tanks se fait sur le modèle polyphonique de l'interview d'experts ou de la citation d'extraits de travaux de chercheurs parlant depuis un institut de recherche désigné comme un think tank. Les chercheurs n'hésitent d'ailleurs pas à instrumentaliser les médias, les journalistes leur servant de porte-voix. «Chaînon manquant 》 entre la décision politique, l'espace public démocratique, les médias et le monde de la recherche, les think tanks sont au cœur de vives tensions communicationnelles. Et si la communication pose autant problème dans les relations d'expertise politiques ou médiatiques, c'est qu'elle 
dévoile le caractère indissociable tout d'abord d'une recherche dialogique d'entente, ensuite de la contrainte des structures institutionnelles, de la pression de systèmes économiques et enfin, de la construction vacillante d'identités hybrides et évolutives, celles de décideur, de journaliste, de chercheur ou d'expert parlant depuis un think tank. Les think tanks se sont institutionnalisés entre être et paraître, fonction sociale et stratégies institutionnelles, illustrant bien la confrontation permanente d'un espoir normatif d'une rationalité dialogique avec une rationalité avant tout procédurale, instrumentale et dramaturgique.
$R \cdot E \cdot F \cdot E \cdot R \cdot E \cdot N \cdot C \cdot E \cdot S$

BADIE Bertrand, HERMET Guy, (1990), La méthode comparative, Paris, Presses Universitaires de France.

BÉLAND Daniel, (2000), «Expertise et politique des retraites: l'influence des think tanks aux États-Unis », in L'Année de la régulation, Dossier Politiques de retraite, $\mathrm{n}^{\circ} 4$.

BOUCHER Stephen (dir.), (2004), L'Europe et ses think tanks : un potentiel inaccompli, Paris, Notre Europe.

CHARAUDEAU Patrick, (2005), Le discours politique. Les masques du pouvoir, Paris, Vuibert.

COHEN Samy, (2003), La résistance des États. Les démocraties face aux défis de la mondialisation, Paris, Seuil.

CROZIER Michel, FRIEDBERG Erhard, (1977), L'acteur et le système : les contraintes de l'action collective, Paris, Seuil.

DICKSON Paul, (1971), Think Tanks, NewYork, Ballantine Books.

DOMHOFF G. William, (1971), « How the Power Elite Set National Goals » in PERRUCCI Robert, PILISUK Mark (dir.), The Triple Revolution Emerging, Boston, Little Brown FIESCHI Catherine, GAFFNEY John (1998), French Think Tanks in Comparative Perspective. pp. 42-58 in STONE Diane, DENHAM Andrew, GARNETT Mark, Think tanks across Nations. A Comparative Approach, Manchester, Manchester University Press.

GAFFNEY John, (1996), « The Political ThinkTanks in the UK and the Ministerial Cabinets in France » in West European Politics, vol. 19, $\mathrm{n}^{\circ} 4$, october. 
GARDIN Jean-Claude, (2004), «La formalisation du discours savant ». [en ligne] Universitat Oberta de Catalunya, [réf. du $1^{\text {er }}$ août 2004]. Disponible sur Internet : http://www.uoc. edu/humfil/digithum/digithum1/gardin/gardin fr_10.htm.

HAMES Tim, FEASEY Richard, (1994), «Anglo-American think tanks under Reagan and Thatcher » in ADONIS Andrew, HAMES Tim (eds.), A conservative revolution? : the Thatcher-Reagan decade in perspective, Manchester, Manchester University Press.

HABERMAS Jürgen, (1999), Morale et communication : conscience morale et activité communicationnelle. Paris, Flammarion.

HAUBERT Maxime et al., (2000), Les sociétés civiles face aux marchés, Paris, Karthala.

JACOBI Daniel, (1986), "Sémiotique du discours de vulgarisation scientifique» in JACOBI Daniel, Diffusion et vulgarisation. Itinéraire du texte scientifique, Paris, Les Belles Lettres.

JAMOUS Haroun, COMMAILLE Jacques, PONS-VIGNON Bernard, (1969), Sociologie de la décision : la réforme des études médicales et des structures hospitalières, Paris, Éd. du CNRS.

LACORNE Denis, RUPNIK Jacques, TOINET Marie-France, (1986), L'Amérique dans les têtes: un siècle de fascinations et d'aversions, Paris, Hachette, 1986.

McGANN James G., (1995), The competition for dollars, scholars and influence in the public policy research industry, Lanbram, University Press of America.

MIGNE Jean, (1969), Le concept de représentation et son rôle dans une pédagogie des connaissances scientifiques, Nancy, INFA, doc R2.

MUCCHIELLI Alex, (1997), "Aux sources de l'influence : la décomposition du processus d'influence» in Médiation, communication et influence. Séminaires thématiques. Études doctorales année universitaire 96-97. Paris, Celsa.

PELLETIER Guy (1995), «Les navigateurs des frontières organisationnelles: regards sur les artisans du partenariat international » in Revue Internationale de Gestion, juin.

PLANTIN Christian, (2004), "Conforts de la polémique" in La langue de la politique: rhétorique de conviction et techniques de communication. $\mathrm{VI}^{\mathrm{e}}$ Forum du Centre international d'études pédagogiques (CIEP), Université Paris 1 Panthéon-Sorbonne.

RIEFFEL Rémy, NEVEU Erik, (1991), "Les effets de réalité des sciences de la communication » in Réseaux, n50, pp. 12-39.

RIEFFEL Rémy, (1993), La tribu des clercs. Les intellectuels sous la V République, 1958-1990, Paris, Calmann-Lévy. CNRS éditions.

ROUBAN Luc, (1993), «L'évaluation, nouvel avatar de la rationalisation administrative? : les limites de l'import-export institutionnel» in Revue française d'administration publique, n66, juin, pp. 197-200.

ROY Olivier, (2001), « Les islamologues ont-ils inventé l'islamisme » in Esprit, août-septembre, pp. 116-138.

STONE Diane, (1996), «From the Margins of Politics: The Influence of Think Tanks in Britain » in West european politics, vol. 19, Oct., ${ }^{\circ} 4$.

TANGUY Lucie, (1995), "Le sociologue et l'expert, une analyse de cas » in Sociologie du travail, $\mathrm{n}^{\circ} 3$, vol. 37, pp. 457-477. 
TAVERNIER Aurélie, (2004), ««Mais d'où ils parlent ?» L'enjeu du titre à parler dans la presse comme lien entre le social et le discursif » in Études de communication, $\mathrm{n}^{\circ} 27$.

TRISTANI-POTTEAUX Françoise, (2001), Du laboratoire au citoyen : les trois étapes de la communication scientifique, CNRS Info, juin, $\mathrm{n}^{\circ} 394$.

TRON George, DENIAU Yves, (2003), «Organismes publics d'évaluation et de prospective économiques et sociales » in Rapport d'information de l'Assemblée nationale, $\mathrm{n}^{\circ} 876$ ( $3^{\mathrm{e}}$ partie). Rapport déposé en application de l'article 145 du Règlement par la Commission des Finances, de l'Économie générale et du plan en conclusion des travaux d'une Mission d'évaluation et de contrôle constituée le 21 janvier 2003. «Organismes publics d'évaluation et de prospective économiques et sociales ». Annexe 2: Les organismes de prospectives à l'étranger, p. 203. http://www.assemblee-nat. fr/12/rap-info/i0876-3.asp

WERNERMichael,ZIMMERMANNBénédicte, (2004), « Penser l'histoire croisée : entre empirie et réflexivité » in De la comparaison à l'histoire croisée, Paris, Seuil, EHESS, nº 42.
$\mathrm{N} \cdot \mathrm{O} \cdot \mathrm{T} \cdot \mathrm{E} \cdot \mathrm{S}$

1. Ce chapitre s'appuie sur une enquête réalisée dans le cadre d'une thèse en Sciences de l'information et de la communication (SIC) : Les limites de « l'importexport institutionnel», le modèle d'expertise anglo-saxon incarné par les think tanks français internationalistes et spécialistes des questions de défense. Plus de 120 entretiens semi-directifs ont ainsi été réalisés entre 2000 et 2004 avec des journalistes, des chercheurs en sciences humaines et sociales ayant réalisé des missions d'expertise pour le ministère des Affaires étrangères ou le ministère de la Défense, et enfin des responsables de la politique extérieure et de défense : ministres, élus nationaux, membres de cabinets ministériels, diplomates, officiers militaires, personnels contractuels divers de l'administration ou et équipes politiques.

2. À propos de l'Ifri [en ligne] Paris : Ifri [Juillet 2004]. Disponible sur Internet :

http://www.ifri.org/frontDispatcher/ifri/a_propos/a propos_de_1_ifri_1046885923539.

3. Erik Izraelewicz, Alain Frachon. «Le premier « think tank » français ». Le Monde, 26.10.1999.

4. Les verbatims de chercheurs, de journalistes et de décideurs rencontrés lors d'entretiens semi-directifs apparaissent dans ce texte de manière non nominative et entre guillemets.

5. Une operating foundation est une fondation qui collecte et gère des fonds mais réalise par elle-même des programmes philanthropiques au lieu de les « sous-traiter ».

6. «I know one when I see one ».

7. L'Institut français des relations internationales (IFRI), le Centre d'études et de recherches internationales (CERI), la Fondation pour la recherche stratégique (FRS), et l'Institut des relations 
internationales et stratégiques (IRIS).

8. Trois titres de la presse quotidienne française de référence ont été dépouillés de façon systématique : Le Monde, Le Figaro et Libération,). Le corpus a été limité dans le temps à une période d'environ six années: 1998-2004. Ces bornes temporelles et la méthode de la recherche par mots-clés ont été choisies en vertu de considérations matérielles : temps de la recherche doctorale, accès aux bases de données et aux versions en ligne de ces quotidiens.

9. Jean-Michel Bezat. «Alain Etchegoyen, un philosophe pour repenser le Plan». Le Monde, 11 avril 2003, p. 17.

10. Serge Michel. « Les Balkans tentent de créer un marché commun pour se rapprocher de l'Europe ». Le Figaro, 18 mars 2003.

11. Jean-Louis Gassé. «Impérial, impérieux, impérialiste? ». Libération, 11 octobre 2002.

12. Keren Lentschner. " $\mathrm{CNN}$ au bord de la crise à la veille d'un conflit majeur ». Le Figaro, 15 janvier 2003.

13. Alain Frachon, Daniel Vernet. « Le stratège et le philosophe ». Le Monde, 12-13, 16 avril 2003.

14. C'est J. R. Goldstein qui s'est ainsi exprimé lors d'un entretien avec Paul Dickson.

15. Rémi Godeau. «La France a encore des atouts, mais... ». Le Figaro, 7 mai 2003.

16. Thomas Lebègue, Vanessa Schneider. «L'UMP, «un an et déjà toute ridée»». Libération, 5 septembre 2003 ; Nicolas Barotte. « L'UMP met en place sa fondation ». Le Figaro, 2 octobre 2003.

17. Béatrice Gurrec. «Le plébiscite de Noël Mamère met fin à l'épisode Alain Lipietz ». Le Monde, 31 Octobre 2001.

18. Christian Losson. «Développement: la Banque mondiale priée de moins pomper». Libération, 11 décembre 2003.

19. Marie Jégo. «Robert Kotcharian a été réélu président de l'Arménie ». Le Monde, 7 mars 2003. 20. Une étude socio-sémiotique des écrits d'écran des think tanks a été complétée par une enquête par entretiens ayant permis de récolter des éléments socio-historiques allant des prémisses de la réflexion en interne sur la mise en ligne de leur site à 2005.

21. Dans Morale et communication, Habermas pose les trois principes suivants : la symétrie des participants à la discussion sans quoi l'argument d'autorité peut survenir, la sincérité des interlocuteurs dans leur désir d'échanger, la liberté d'adhésion c'est-à-dire l'absence de contrainte extérieure.

22. Aux États-Unis, le titre à parler le plus courant est celui du diplôme. Même pour introduire le plus réputé des chercheurs travaillant pour le plus célèbre des think tanks, il sera fait mention de son niveau de diplôme. Son nom sera précédé ou bien suivi de la mention : Dr., ou PhD. 
$R \cdot E \cdot S \cdot U \cdot M \cdot E ́$

Centré sur les effets d'information et d'image en politique, cet article interroge tout d'abord les enjeux politiques des débats théoriques portant sur la définition et le label «think tanks ». Les stratégies de communication externe des think tanks français spécialistes des relations internationales et des questions de défense ont répondu à une crise matérielle (pérennité institutionnelle) et identitaire (visibilité et légitimité). Les discours sur les think tanks français oscillent en effet entre dénégation et affirmation de leurs compétence, indépendance, transparence, et utilité politique et sociale. Les outils d'information et de communication des think tanks français connaissent un double circuit, expertal et médiatique. Cet article questionne donc les relations ambiguës qui se nouent via les think tanks entre trois pôles : des « décideurs » aux motivations complexes; des journalistes avides de caution scientifique ; et des « chercheurs » bénéficiant des profits matériels et symboliques d'une expression depuis un think tank.

This paper examines concepts such as information, image, identity, legitimacy and instrumentalisation in the field of French public policy expertise. Speaking of French "think tanks" implies that some institutions can be referred to as such whereas the Anglo-American "think tank" denomination refers to subjective interpretations and positive images and values such as stability, financial autonomy, competence, visibility, legitimate participation in the decision making processes, building of a well informed civil society. Communication strategies of French think tanks dealing with international relations and strategic issues are aimed to get the "think tank label" and to contradict criticisms and denegation. Researchers produce and spread informations and analysis through a double cir- cuit: they work as consultants for administrative bodies and for journalists. French think tanks officially fulfill an increasing demand for public policy advice. Above all, they contribute to creating a positive image for decision-makers (wise and well advised) and journalists (well informed) and they allow some researchers to enjoy a stable position and a prestigious status. 
